Title: $\quad$ Fitting of the Data for Diffusion Coefficients in Unsaturated Porous Media

Document Identifier: B00000000-01717-0210-00026 REV 00

Originator:

Checker:

Lead Discipline Engineer:

Draft Date:
Bryan E. Bullard

Bryan Dunlap

David Sevougian

August 6, 1998 
Title: Fitting of the Data for Diffusion Coefficients in Unsaturated Porous Media

\section{Table of Contents:}

$\underline{\text { Item }}$

1.0 Purpose

4

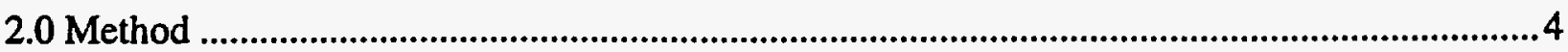

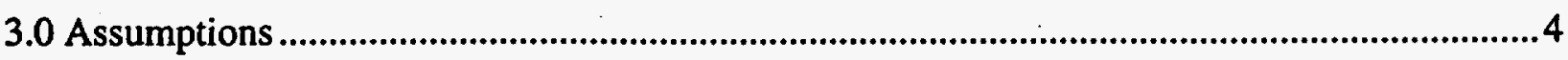

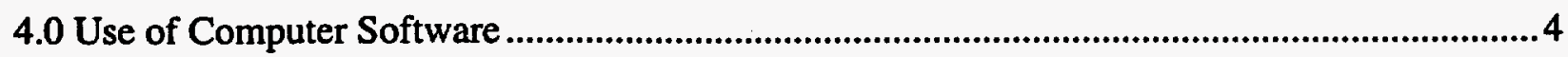

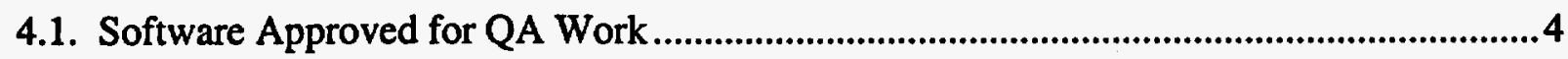

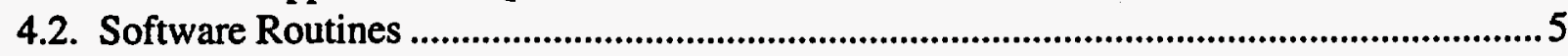

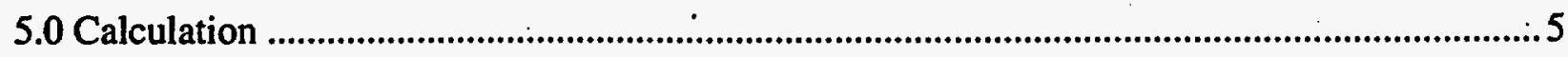

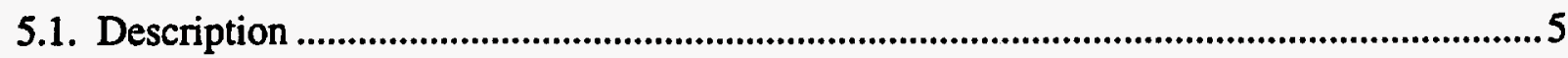

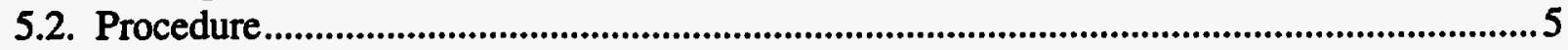

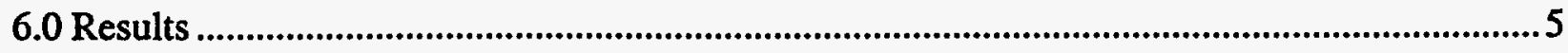

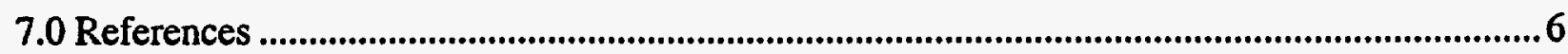

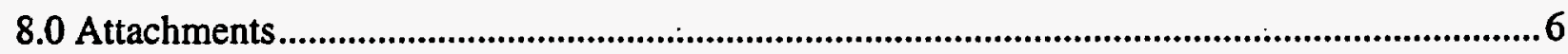


Title: Fitting of the Data for Diffusion Coefficients in Unsaturated Porous Media

Document Identifier: B00000000-01717-0210-00026 REV 00 (August 6, 1998)

Page 4 of 6

\subsection{Purpose}

The purpose of this calculation is to evaluate diffusion coefficients in unsaturated porous media for use in the TSPA-VA analyses. Using experimental data, regression techniques were used to curve fit the diffusion coefficient in unsaturated porous media as a function of volumetric water content. This calculation substantiates the model fit used in Total System Performance Assessment-1995 An Evaluation of the Potential Yucca Mountain Repository (TSPA-1995), Section 6.5.4.

\subsection{Method}

Using an unsaturated flow apparatus based on open-flow centrifugation, Conca and co-workers at Pacific Northwest National Laboratories measured aqueous diffusion coefficients in a wide spectrum of geologic materials at varying degrees of water saturation. It was suggested from their study that diffusion coefficients are functions primarily of water content and not materials characteristics. The data set and model used were as given in TSPA-1995, Section 6.5.4. The model was fit by a least squares method. The functional form is expressed as

$$
\log D_{p}=a+b \log \phi+\varepsilon
$$

Where $D_{p}$ is the diffusion coefficient $\left(\mathrm{cm}^{2} / \mathrm{sec}\right)$ and $\phi$ is the volumetric water content in percent and $\varepsilon$ is random error. The model parameters $a$ and $b$ are determined from the least squares fit. The data set (DTN: MO9807SPA00026.000) was supplied from Engel (1995). The data is not qualified and results derived from it should be considered "to be verified" under TBV-377. A general discussion related to the data set can be found in Conca and Wright (1992).

\subsection{Assumptions}

The following assumptions were made:

1. The model predictions assume that the log base 10 transformed output variables have identical error distributions and that these error distributions are normally distributed. The basis of this assumption is supported by the data gathering method and analysis of the residuals. This assumption is used throughout the calculation.

2. The model as given in TSPA-1995 is assumed to be of the proper functional form. This assumption is used throughout the calculation.

\subsection{Use of Computer Software}

\subsection{Software Approved for $Q A$ Work} N/A. 
Title: Fitting of the Data for Diffusion Coefficients in Unsaturated Porous Media Document Identifier: B00000000-01717-0210-00026 REV 00 (Auguss 6, 1998)

Page 5 of 6

\subsection{Software Routines}

The software used was Mathcad 7 Professional. The type of computer used was a Dell PowerEdge 2200 with a Pentium II - $266 \mathrm{MHz}$ CPU, $128 \mathrm{MB}$ of RAM, in a Windows NT 4.0 operating system.

Mathcad 7 is an appropriate application because it provides a wide variety of mathematical functions that are necessary for the calculations used. Also, the program provides ease of display of equations and rationale employed, which eliminates the need to attach extra explanation and justification for the specific methodology.

\subsection{Calculation}

All calculations use data (DTN: MO9807SPA00026.000) contained in Engel (1995). This input data is "to be verified" (TBV-377) and thus, the results of this calculation should be considered "to be verified."

\subsection{Description}

The calculation inputs consist of 177 observations of diffusion coefficients for various degrees of water saturation, the source of the data is shown in Attachment II, Engel (1995), (values are also shown in Attachment I, Section B - Data Listing on pages I-4 to I-10).

See Attachment I, Section A for a further description of the calculation.

\subsection{Procedure}

See Attachment I, Section A.

\subsection{Results}

All results are to be considered "to be verified" (TBV-377) as this calculations uses as inputs data (DTN: M09807SPA00026.000) contained in Engel (1995). A predicted value of an individual observation will be given by the following equation and standard deviation below

$$
\begin{aligned}
& \log D_{p}=a+b \log \phi+z \cdot \operatorname{std}(\phi) \\
& \operatorname{std}(\phi)=\sqrt{\Sigma_{00}+2 \cdot \Sigma_{01} \cdot \log (\phi)+\Sigma_{11} \cdot \log (\phi)^{2}+\sigma_{\varepsilon}{ }^{2}}
\end{aligned}
$$

Where $z$ is a standard normal, $D_{p}$ is the diffusion coefficient $\left(\mathrm{cm}^{2} / \mathrm{sec}\right)$ and $\phi$ is the volumetric water content in percent. Numerical values for the model parameters from the regression fit are:

$$
a=-8.254664, \mathrm{~b}=1.89762, \sigma_{\varepsilon}{ }^{2}=0.086845, \Sigma=\left[\begin{array}{cc}
2.468868 \mathrm{E}-3 & -2.052563 \mathrm{E}-3 \\
-2.052563 \mathrm{E}-3 & 2.129701 \mathrm{E}-3
\end{array}\right]
$$

For further details see Attachment I, Section A. 
Title: Fitting of the Data for Diffusion Coefficients in Unsaturated Porous Media

Document Identifier: B00000000-01717-0210-00026 REV 00 (August 6. 1998)

Page 6 of 6

\subsection{References}

Conca, J. L., and J. Wright, 1992. "Diffusion and Flow in Gravel Soil, and Whole Rock," Applied Hydrogeology, Vol. 1, pp. 5-24. TIC\#: 224081.

Engel, D., 1995 Memo to J.H. Lee, January 30. DTN: MO90807SPA00026.000. TBV-377.

M\&O (Civilian Radioactive Waste Management System, Management and Operating Contractor [CRWMS M\&O]), 1995. Total System Performance Assessment-1995: An Evaluation of the Potential Yucca Mountain Repository, B00000000-01717-2200-00136, Rev. 01, Las Vegas, NV. MOL.19960724.0188.

\subsection{Attachments}

\section{ATTACHMENT}

TITLE

Diffusion Coefficient in Unsaturated Porous Media

Memo to J.H. Lee, January 30
NUMBER OF

PAGES

11 


\section{Attachment I - Diffusion Coefficient in Unsaturated Porous Media}

\section{Section A - Regression Calculation}

Measured diffusion coefficients in a geologic materials at varying degrees of water saturation show a functional relationship between the diffusion coefficient in unsaturated porous media and the volumetric water content.

The following data DTN: MO9807SPA00026.000 (input file data3) originates from an e-mail, Attachment II (Engel, 1995). The data matrix is displayed in Section B - Data Listing on pages I-5 to 1-11. Four points have been removed consisting of the data set labeled benton/sand, density=2.00. The data is not qualified and all results are "to be verified" under TBV-377.

$$
\text { data }:=\quad \text { D:Idiffusldata3 bentonsand }:=\left[\begin{array}{cc}
1.3 \cdot 10^{1} & 5 \cdot 10^{-8} \\
1.3 \cdot 10^{1} & 4 \cdot 10^{-8} \\
1.3 \cdot 10^{1} & 1.6 \cdot 10^{-8} \\
1.3 \cdot 10^{1} & 3 \cdot 10^{-9}
\end{array}\right] \begin{aligned}
& \text { Points removed from } \\
& \text { regression analysis, see } \\
& \text { graph below. }
\end{aligned}
$$

rows $($ data $)=177 \quad \operatorname{cols}($ data $)=2 \quad 177$ data pairs were used in the regression fit.

i :=0.. rows(data) $-1 \quad$ row index for values in data matrix, indexing starts with zero.

$\mathbf{y}_{\mathbf{i}}:=\log \left(\right.$ data $\left._{\mathrm{i}, 1}\right)$

$x_{i}:=\log \left(\right.$ data $\left._{i, 0}\right)$
- y values are $\log 10(D p)$, where $D p$ is pore diffusion in $\mathrm{cm}^{2} / \mathrm{s}$

- $x$ values are $\log 10(\phi)$, where $\phi$ is percent volumetric water content

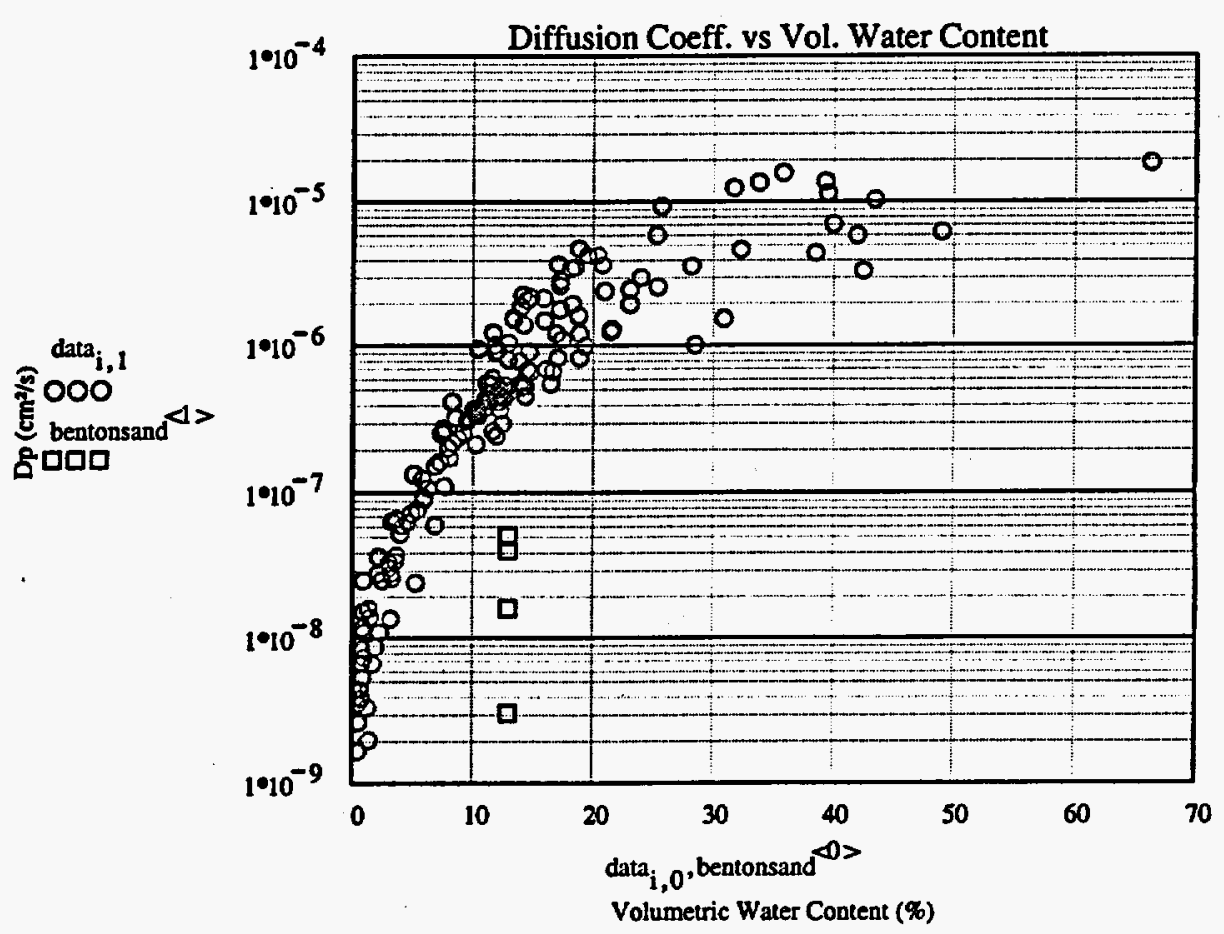


Create system of equations of form $y=a+b x$ to solve in matrix form

$X_{1,0}:=1 \quad X_{1,1}:=x_{i}$

invXX := $\left(X^{T} \cdot X\right)^{-1}$

$B:=\operatorname{invXX} \cdot X^{T} \cdot y \quad B=\left[\begin{array}{l}-8.254664 \\ 1.89762\end{array}\right]$

verification using built in functions

$$
\begin{aligned}
& \operatorname{intercept}(x, y)=-8.254664 \\
& \operatorname{slope}(x, y)=1.89762
\end{aligned}
$$

$\mathrm{a}:=\mathrm{B}_{0,0} \quad \mathrm{~b}:=\mathrm{B}_{1,0}$ $\sigma \varepsilon 2:=\frac{\left(\mathrm{y}^{\mathrm{T}} \cdot \mathrm{y}-\mathrm{B}^{\mathrm{T}} \cdot \mathrm{X}^{\mathrm{T}} \cdot \mathrm{y}\right)}{(\operatorname{rows}(\mathrm{X})-1)} \quad \begin{aligned} & \text { Estimate of the residual variance. Note that for an unbiased } \\ & \text { estimator the denominator term should be minus two not one, the }\end{aligned}$

$\sigma \varepsilon 2=0.086845$ used in TSPA-VA. This change alters the result in the error variance at the fourth decimal place and the fifth decimal place for $\Sigma:=\sigma \varepsilon 2_{0,0} \cdot \operatorname{invXX}$ the variance-covariance matrix. (Corrected values are calculated $\Sigma=\left[\begin{array}{cc}2.468868 \cdot 10^{-3} & -2.052563 \cdot 10^{-3} \\ -2.052563 \cdot 10^{-3} & 2.129701 \cdot 10^{-3}\end{array}\right] \quad \begin{aligned} & \text { - Estimate of the variance-covariance matrix for } \\ & \text { coefficient vector } \mathrm{B} \text {. }\end{aligned}$ $\operatorname{Syo}(x):=\sqrt{\Sigma_{0,0}+2 \cdot \Sigma_{0,1} \cdot x+\Sigma_{1,1} \cdot x^{2}+\sigma \varepsilon 2_{0,0}}$ value of y given at $x$. Standard deviation for individual predicted yo $(x, z):=a+b \cdot x+z \cdot \operatorname{Syo}(x)$

$\mathrm{n}:=60$ high $:=\max (\mathrm{x})$ low $:=\min (\mathrm{x}) \quad$ ii $:=0 . . \mathrm{n}-1 \quad \mathrm{xx}_{\mathrm{ii}}:=\mathrm{low}+\left(\frac{\mathrm{high}-\mathrm{low}}{\mathrm{n}-1}\right) \cdot \mathrm{ii}$

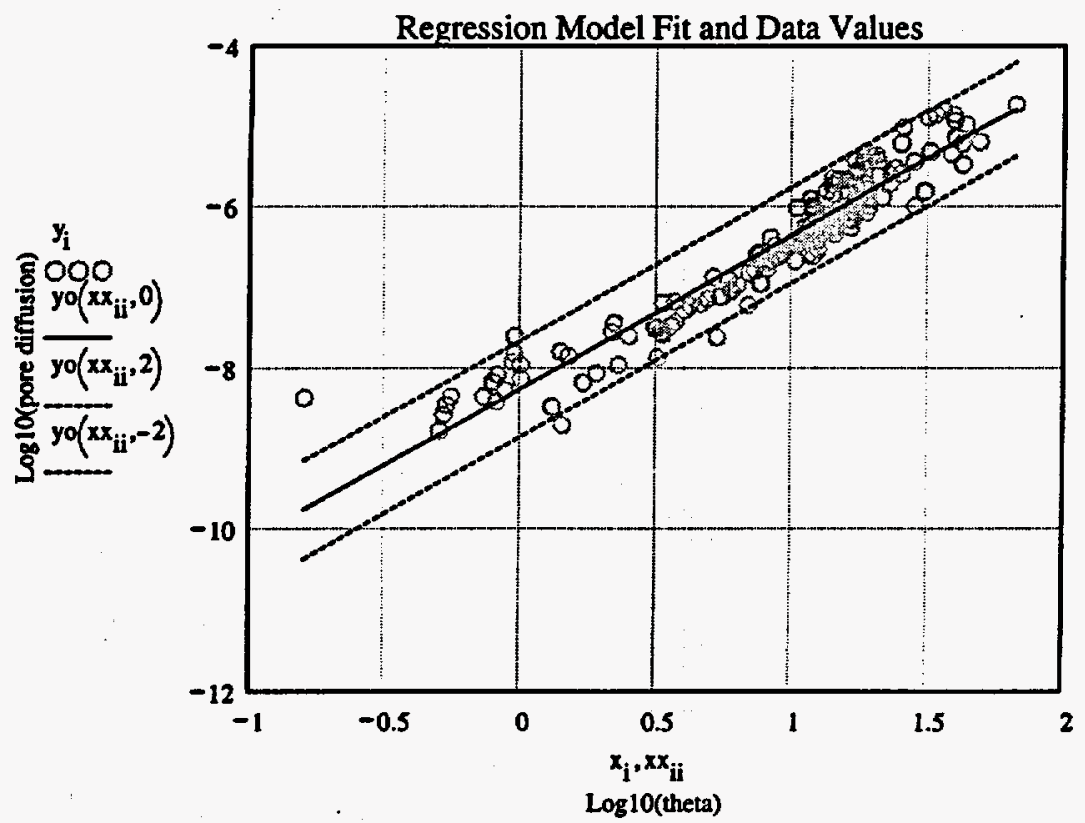


- Note one point (1.6e-1, 4.31e-9) appears as an outlier at the lower limit of percent volumetric water content. Solid line is mean value and dashed lines are \pm 2 std.

pow $10(y):=10^{y} \quad$ - Create a function to raise argument to power base 10.

Compare below with Figure 6.5-6 in TSPA-1995, page 6-55

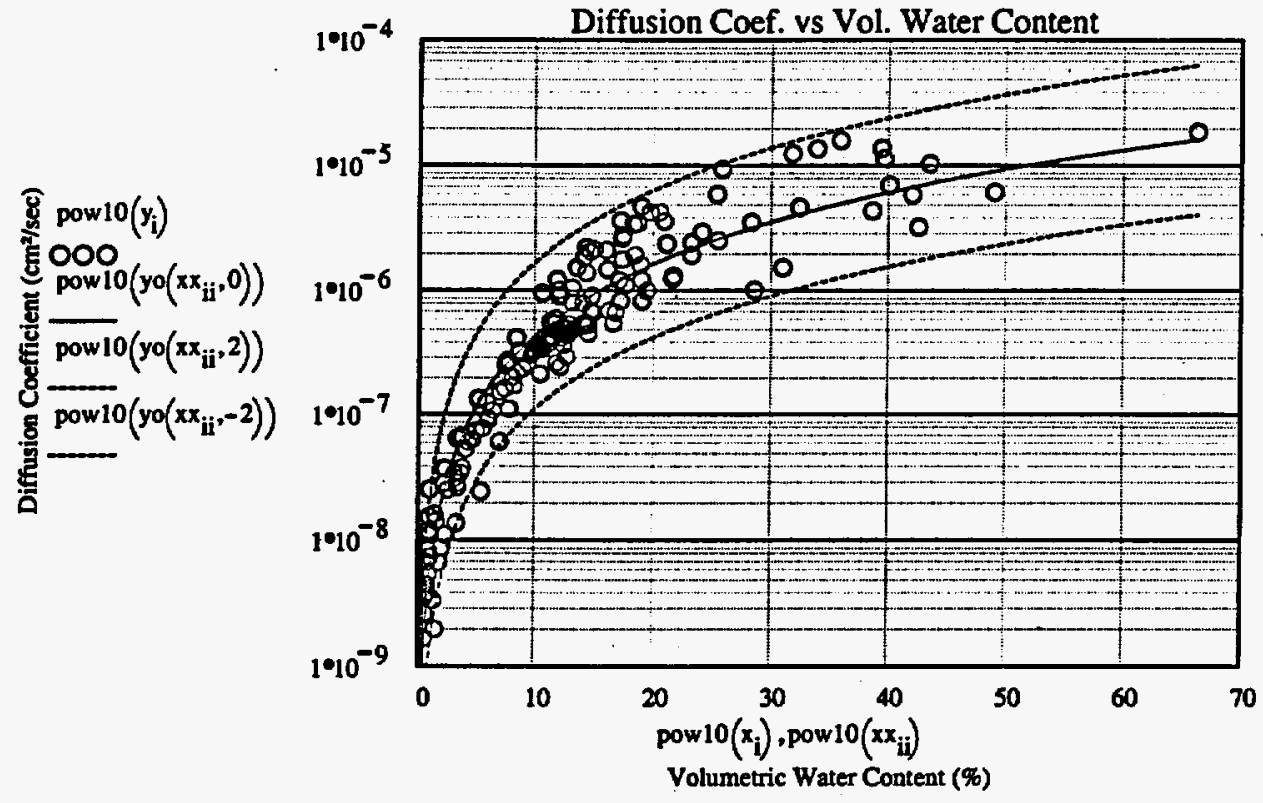

- Model Estimation and the Uncertainty for the Diffusion Coefficient in Porous Geologic Media as a Function of the Volumetric Water Content. The raw data for a suite of geological materials were kindly provided by Jim Conce through David Engel at PNL (Engel, 1995).

Summary

$a=-8.254664 \quad b=1.89762 \quad \sigma \varepsilon 2=0.086845 \quad \Sigma=\left[\begin{array}{cc}2.468868 \cdot 10^{-3} & -2.052563 \cdot 10^{-3} \\ -2.052563 \cdot 10^{-3} & 2.129701 \cdot 10^{-3}\end{array}\right]$

Pore diffusivity and effective diffusion coefficient (Deff) in $\mathrm{m}^{2} / \mathrm{s}$ given $\phi$ and a standard normal value, $z$. Note: $y$ values are $\log 10(D p)$, where $D p$ is pore diffusion in $\mathrm{cm}^{2} / \mathrm{s}$. $x$ values are $\log 10\langle\phi\rangle$, where $\phi$ is percent volumetric water content.

$$
\begin{aligned}
& \operatorname{Syo}(x):=\sqrt{\Sigma_{0,0}+2 \cdot \Sigma_{0,1} \cdot x+\Sigma_{1,1} \cdot x^{2}+\sigma \varepsilon 2_{0,0}} \\
& \operatorname{yo}(x, z):=a+b \cdot x+z \cdot \operatorname{Syo}(x) \\
& \operatorname{Dpore}(\phi, z):=\operatorname{pow} 10(\operatorname{yo}(\log (\phi), z)) \cdot 10^{-4} \\
& \operatorname{Deff}(\phi, z):=\frac{\phi \cdot \operatorname{Dpore}(\phi, z)}{100} .
\end{aligned}
$$




\section{Corrected values in the calculation of the residual variance}

The $a$ and $b$ coefficient values remain unchanged.

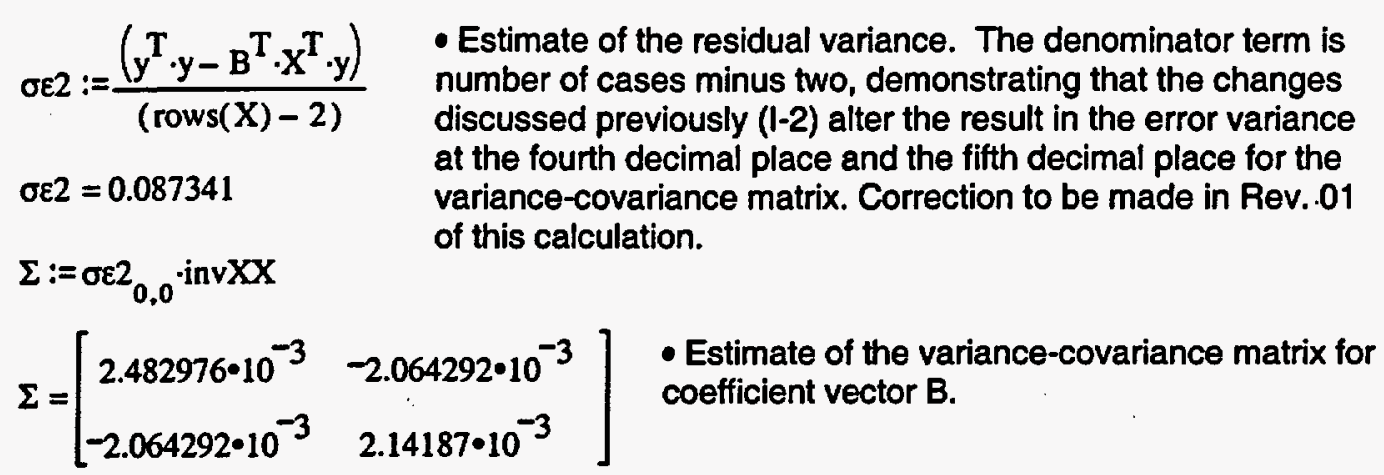

$\operatorname{Syo}(x):=\sqrt{\Sigma_{0,0}+2 \cdot \Sigma_{0,1} \cdot x+\Sigma_{1,1} \cdot x^{2}+\sigma \varepsilon 2_{0,0}}$ - Standard deviation for an individual predicted $\operatorname{yo}(x, z):=a+b \cdot x+z \cdot \operatorname{Syo}(x)$

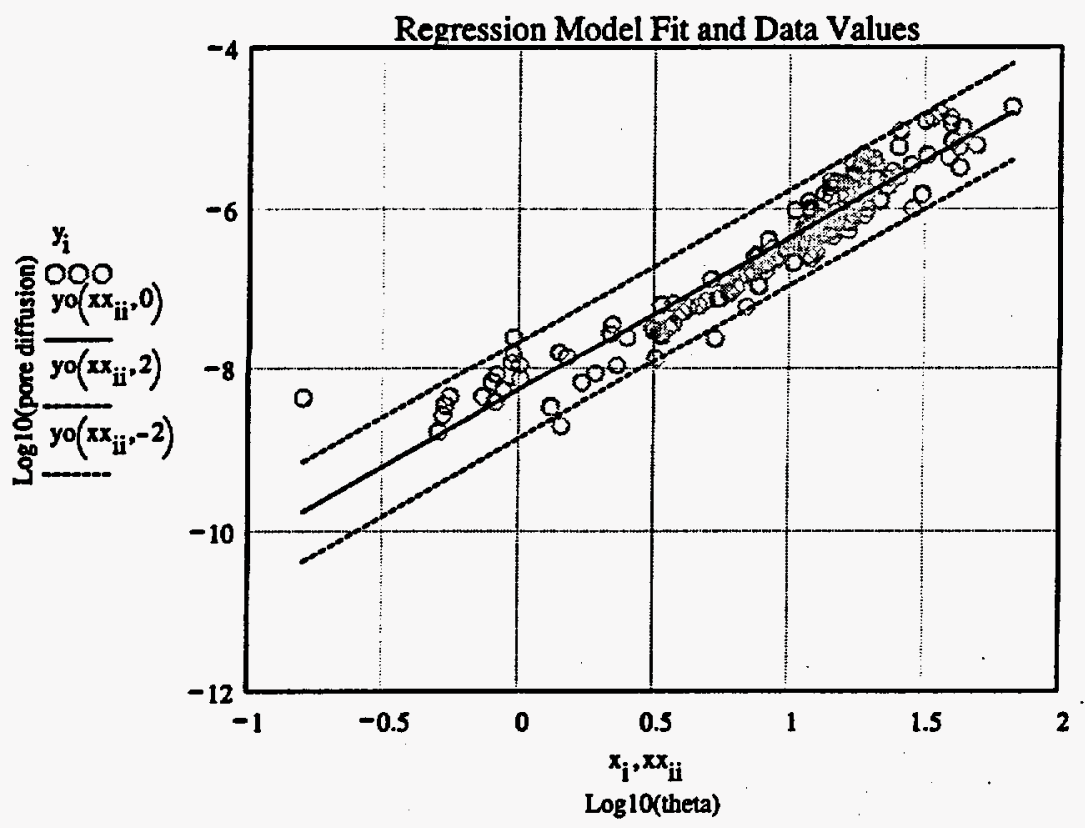

- Note one point (1.6e-1, 4.31e-9) appears as an outlier at the lower limit of percent volumetric water content. Solid line is mean value and dashed lines are \pm 2 std. 
Section B - Data Listing (DTN: MO9807SPA00026.000)

Volumetric Water Pore Diffusion Content (\%) data $=$ $\left[\begin{array}{l}12 . \\ 11 \\ 10 \\ 8.800 \\ 8.000 \\ 7.300\end{array}\right.$
Data is "to be verified" and all results based on this dat set should be tracked with TBV-377.

$$
\begin{array}{ll}
28.200000 & 3.500000 \cdot 10^{-6} \\
32.300000 & 4.600000 \cdot 10^{-6} \\
11.760000 & 4.800000 \cdot 10^{-7} \\
8.320000 & 4.100000 \cdot 10^{-7} \\
23.100000 & 2.400000 \cdot 10^{-6} \\
16.700000 & 6.600000 \cdot 10^{-7} \\
13.900000 & 7.800000 \cdot 10^{-7} \\
11.600000 & 5.400000 \cdot 10^{-7} \\
8.600000 & 3.200000 \cdot 10^{-7} \\
7.600000 & 2.600000 \cdot 10^{-7} \\
7.400000 & 2.500000 \cdot 10^{-7} \\
25.400000 & 2.500000 \cdot 10^{-6} \\
23.100000 & 1.900000 \cdot 10^{-6} \\
18.900000 & 1.200000 \cdot 10^{-6} \\
17.500000 & 1.100000 \cdot 10^{-6} \\
17.000000 & 1.200000 \cdot 10^{-6} \\
24.000000 & 2.900000 \cdot 10^{-6} \\
21.000000 & 2.340000 \cdot 10^{-6} \\
18.300000 & 1.900000 \cdot 10^{-6} \\
17.300000 & 1.760000 \cdot 10^{-6} \\
&
\end{array}
$$$$
\text { - Start of Data Set } 1
$$ 


$\mid \begin{array}{ll}16.000000 & 1.470000 \cdot 10^{u} \\ 18.800000 & 1.600000 \cdot 10^{-6} \\ 17.100000 & 8.200000 \cdot 10^{-7} \\ 12.000000 & 2.400000 \cdot 10^{-7} \\ 14.700000 & 9.000000 \cdot 10^{-7} \\ 14.400000 & 5.200000 \cdot 10^{-7} \\ 8.100000 & 1.700000 \cdot 10^{-7} \\ 5.900000 & 9.300000 \cdot 10^{-8} \\ 4.900000 & 7.200000 \cdot 10^{-8} \\ 7.700000 & 1.100000 \cdot 10^{-7} \\ 5.830000 & 1.230000 \cdot 10^{-7} \\ 5.400000 & 7.600000 \cdot 10^{-8} \\ 3.570000 & 3.370000 \cdot 10^{-8} \\ 6.900000 & 6.000000 \cdot 10^{-8} \\ 3.340000 & 2.600000 \cdot 10^{-8} \\ 3.700000 & 3.700000 \cdot 10^{-8} \\ 5.300000 & 2.400000 \cdot 10^{-8} \\ 14.400000 & 4.500000 \cdot 10^{-7} \\ 12.500000 & 2.900000 \cdot 10^{-7} \\ 12.300000 & 4.400000 \cdot 10^{-7} \\ 11.700000 & 2.600000 \cdot 10^{-7} \\ 12.300000 & 3.600000 \cdot 10^{-7} \\ 10.300000 & 2.100000 \cdot 10^{-7} \\ 11.200000 & 5.480000 \cdot 10^{-7} \\ 7.600000 & 1.100000 \cdot 10^{-7} \\ 3.330000 & 6.350000 \cdot 10^{-8} \\ 3.140000 & 3.060000 \cdot 10^{-8} \\ 2.290000 & 1.090000 \cdot 10^{-8} \\ 1.420000 & 1.950000 \cdot 10^{-9}\end{array}$




$\mid$\begin{tabular}{cc}
7.600000 & $2.690000 \cdot 10^{-7}$ \\
6.930000 & $1.500000 \cdot 10^{-7}$ \\
5.510000 & $7.680000 \cdot 10^{-8}$ \\
3.270000 & $2.790000 \cdot 10^{-8}$ \\
3.200000 & $1.350000 \cdot 10^{-8}$ \\
40.000000 & $6.900000 \cdot 10^{-6}$ \\
3.700000 & $6.600000 \cdot 10^{-8}$ \\
2.200000 & $3.630000 \cdot 10^{-8}$ \\
1.000000 & $1.090000 \cdot 10^{-8}$ \\
0.810000 & $3.800000 \cdot 10^{-9}$ \\
5.100000 & $1.320000 \cdot 10^{-7}$ \\
1.500000 & $1.390000 \cdot 10^{-8}$ \\
1.000000 & $7.300000 \cdot 10^{-9}$ \\
0.730000 & $4.400000 \cdot 10^{-9}$ \\
4.200000 & $5.940000 \cdot 10^{-8}$ \\
1.700000 & $6.600000 \cdot 10^{-9}$ \\
1.300000 & $3.300000 \cdot 10^{-9}$ \\
4.000000 & $5.220000 \cdot 10^{-8}$ \\
49.000000 & $6.090000 \cdot 10^{-6}$ \\
0.500000 & $1.650000 \cdot 10^{-9}$ \\
0.520000 & $2.600000 \cdot 10^{-9}$ \\
0.530000 & $3.480000 \cdot 10^{-9}$ \\
0.550000 & $4.420000 \cdot 10^{-9}$ \\
0.790000 & $6.660000 \cdot 10^{-9}$ \\
0.930000 & $1.180000 \cdot 10^{-8}$ \\
1.400000 & $1.590000 \cdot 10^{-8}$ \\
2.170000 & $2.770000 \cdot 10^{-8}$ \\
\hline .000000 & $8.920000 \cdot 10^{-8}$ \\
\hline
\end{tabular}




$\left|\begin{array}{ll}4.600000 & 6.210000 \cdot 10^{0} \\ 3.100000 & 3.300000 \cdot 10^{-8} \\ 2.500000 & 2.500000 \cdot 10^{-8} \\ 1.900000 & 8.600000 \cdot 10^{-9} \\ 6.300000 & 1.060000 \cdot 10^{-7} \\ 38.540000 & 4.334000 \cdot 10^{-6} \\ 10.050000 & 3.510000 \cdot 10^{-7} \\ 10.210000 & 3.540000 \cdot 10^{-7} \\ 11.130000 & 4.270000 \cdot 10^{-7} \\ 12.720000 & 5.320000 \cdot 10^{-7} \\ 14.580000 & 6.820000 \cdot 10^{-7} \\ 21.590000 & 1.288000 \cdot 10^{-6} \\ 10.260000 & 3.340000 \cdot 10^{-7} \\ 11.190000 & 4.190000 \cdot 10^{-7} \\ 12.020000 & 4.470000 \cdot 10^{-7} \\ 12.320000 & 4.500000 \cdot 10^{-7} \\ 13.070000 & 4.770000 \cdot 10^{-7} \\ 13.880000 & 5.390000 \cdot 10^{-7} \\ 14.170000 & 5.520000 \cdot 10^{-7} \\ 16.140000 & 6.820000 \cdot 10^{-7} \\ 9.240000 & 2.550000 \cdot 10^{-7} \\ 9.640000 & 3.070000 \cdot 10^{-7} \\ 10.350000 & 3.400000 \cdot 10^{-7} \\ 11.370000 & 4.270000 \cdot 10^{-7} \\ 9.240000 & 2.550000 \cdot 10^{-7} \\ \hline .560000 & 3.000000 \cdot 10^{-7} \\ \hline 1600000 & 3.300000 \cdot 10^{-7} \\ 4.090000 \cdot 10^{-7} \\ 5.120000 \cdot 10^{-7}\end{array}\right|$




\begin{tabular}{|c|c|}
\hline 18.920000 & $8.190000 \cdot 10^{-7}$ \\
\hline 10.910000 & $3.620000 \cdot 10^{-7}$ \\
\hline 11.400000 & $4.120000 \cdot 10^{-7}$ \\
\hline 12.690000 & $4.370000 \cdot 10^{-7}$ \\
\hline 14.360000 & $4.500000 \cdot 10^{-7}$ \\
\hline 16.520000 & $5.450000 \cdot 10^{-7}$ \\
\hline 21.530000 & $1.232000 \cdot 10^{-6}$ \\
\hline 9.750000 & $3.200000 \cdot 10^{-7}$ \\
\hline 10.110000 & $3.620000 \cdot 10^{-7}$ \\
\hline 11.100000 & $4.220000 \cdot 10^{-7}$ \\
\hline 12.270000 & $5.050000 \cdot 10^{-7}$ \\
\hline 14.820000 & $6.540000 \cdot 10^{-7}$ \\
\hline 19.370000 & $9.890000 \cdot 10^{-7}$ \\
\hline 8.350000 & $2.150000 \cdot 10^{-7}$ \\
\hline 20.800000 & $3.580000 \cdot 10^{-6}$ \\
\hline 33.800000 & $1.344000 \cdot 10^{-5}$ \\
\hline 35.800000 & $1.568000 \cdot 10^{-5}$ \\
\hline 25.300000 & $5.824000 \cdot 10^{-6}$ \\
\hline 31.700000 & $1.232000 \cdot 10^{-5}$ \\
\hline 20.390000 & $4.194400 \cdot 10^{-6}$ \\
\hline 25.700000 & $9.259040 \cdot 10^{-6}$ \\
\hline 43.440000 & $1.020320 \cdot 10^{-5}$ \\
\hline 39.320000 & $1.360800 \cdot 10^{-5}$ \\
\hline 39.530000 & $1.131200 \cdot 10^{-5}$ \\
\hline 66.300000 & $1.830000 \cdot 10^{-5}$ \\
\hline 30.900000 & $1.510000 \cdot 10^{-6}$ \\
\hline 28.500000 & $1.000000 \cdot 10^{-6}$ \\
\hline 42.500000 & $3.220000 \cdot 10^{-6}$ \\
\hline
\end{tabular}




$\left|\begin{array}{ll}13.000000 & 7.940000 \cdot 10^{-1} \\ 41.960000 & 5.800000 \cdot 10^{-6} \\ 0.949000 & 2.500000 \cdot 10^{-8} \\ 0.160000 & 4.310000 \cdot 10^{-9} \\ 0.819000 & 8.300000 \cdot 10^{-9} \\ 17.320000 & 2.755200 \cdot 10^{-6} \\ 33.800000 & 1.344000 \cdot 10^{-5} \\ 35.800000 & 1.568000 \cdot 10^{-5} \\ 25.300000 & 5.824000 \cdot 10^{-6} \\ 31.700000 & 1.232000 \cdot 10^{-5} \\ 15.920000 & 2.083200 \cdot 10^{-6} \\ 17.270000 & 2.564800 \cdot 10^{-6} \\ 18.190000 & 3.363360 \cdot 10^{-6} \\ 18.540000 & 3.438400 \cdot 10^{-6} \\ 19.600000 & 4.194400 \cdot 10^{-6} \\ 20.390000 & 4.194400 \cdot 10^{-6} \\ 14.240000 & 1.383200 \cdot 10^{-6} \\ 11.650000 & 5.895700 \cdot 10^{-7} \\ 14.540000 & 2.027200 \cdot 10^{-6} \\ 17.110000 & 3.595200 \cdot 10^{-6} \\ 18.850000 & 4.681600 \cdot 10^{-6} \\ 12.050000 & 9.221000 \cdot 10^{-7} \\ 14.180000 & 2.191840 \cdot 10^{-6} \\ 11.960000 & 8.880500 \cdot 10^{-7} \\ 13.000000 & 1.036800 \cdot 10^{-6} \\ 14.060000 & 1.828960 \cdot 10^{-6} \\ 25.700000 & 9.259040 \cdot 10^{-6} \\ 430000 & 1.020320 \cdot 10^{-5} \\ \hline 320000 & 1.360800 \cdot 10^{-5}\end{array}\right|$

-Where benton/sand was removed

- Data pair labeled Japanese cores mudstone (1.6e-1 4.31e-9). Outlier very close to $0.1 \%$ limit, suspect value.

- Start of Data Set 2.

Starting with pair (17.32, .027552e-4) 


$\left|\begin{array}{ll}39.530000 & 1.131200 \cdot 10^{-5} \\ 0.870000 & 5.290000 \cdot 10^{-9} \\ 0.945000 & 1.510000 \cdot 10^{-8} \\ 14.900000 & 2.120000 \cdot 10^{-6} \\ 13.440000 & 1.510880 \cdot 10^{-6} \\ 11.910000 & 1.001390 \cdot 10^{-6} \\ 11.730000 & 1.210000 \cdot 10^{-6} \\ 10.480000 & 9.447000 \cdot 10^{-7}\end{array}\right|$


Memo to J.H. Lee, January 30

Engel, D., 1995

- Electronic memo from Engel to Lee January 30, 1995.

- Data Set 2 is in different units than Data Set 1. Units were inferred from TSPA-1995 analysis.

- Data has been assigned data tracking number (DTN: M09807SPA00026.000).

- Not qualified data, results based on this data are "to be verified" under (TBV-377).

- Text of email follows:

To: Joon Lee

cC:

Subject: $\quad$ Re: Jim's D(eff) Raw Data

Joon,

Below are the data sets from Jim Conca. I did not have all of the information that you requested per data point, but this is what we got from Jim.

The first set contains volumetric water content and diff coeff. for different samples. The second set contain volumetric water content. diff coeff., and density for compacted bentonite. I will fax you a description of that data.

If you have any questions, please call,

Dave Engel

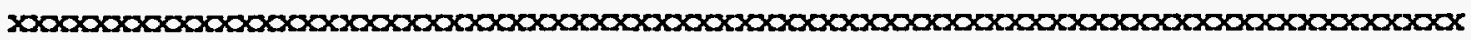

Data Set 1

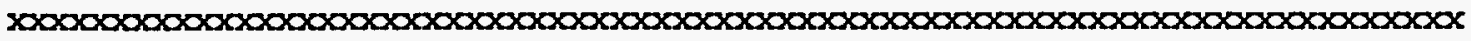

Attached is the diffusion coefficient versus water content data (Figure 2) from Diffusion and Flow in Gravel, Soil, and Whole Rock by Jim Conca and Judith Wright (Applied Hydrology 1/92)

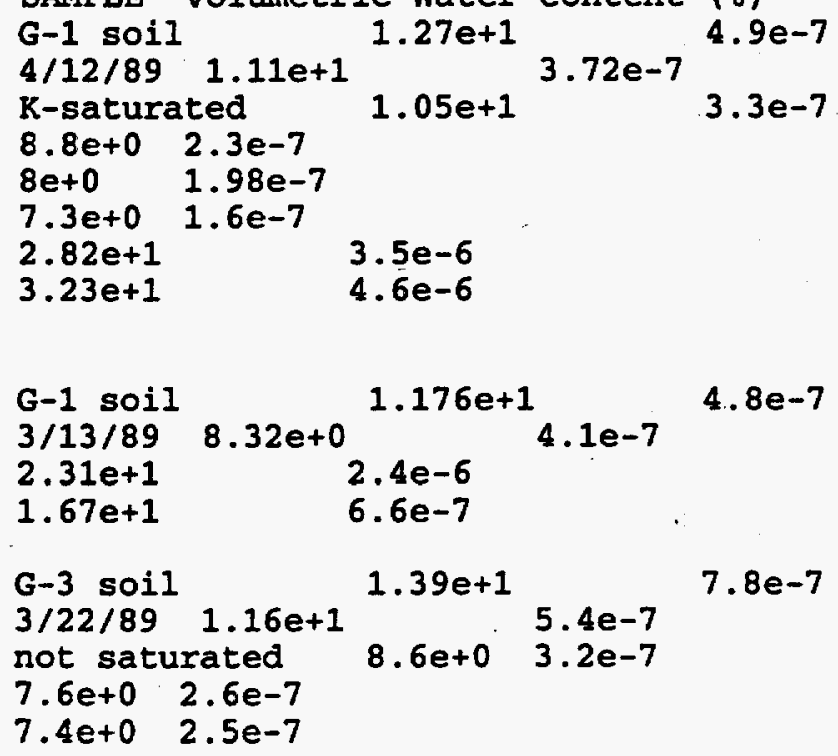


Memo to J.H. Lee, January 30.txt

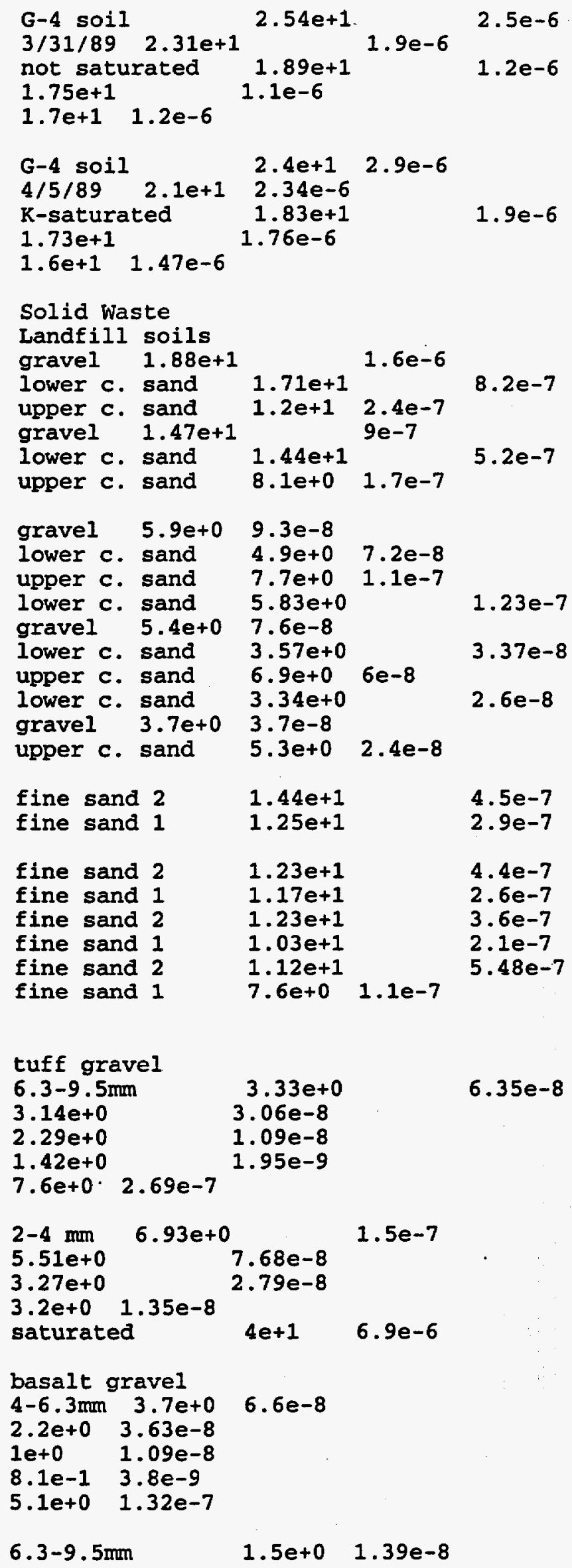


Memo to J.H. Lee, January 30. txt $8 / 3 / 98$

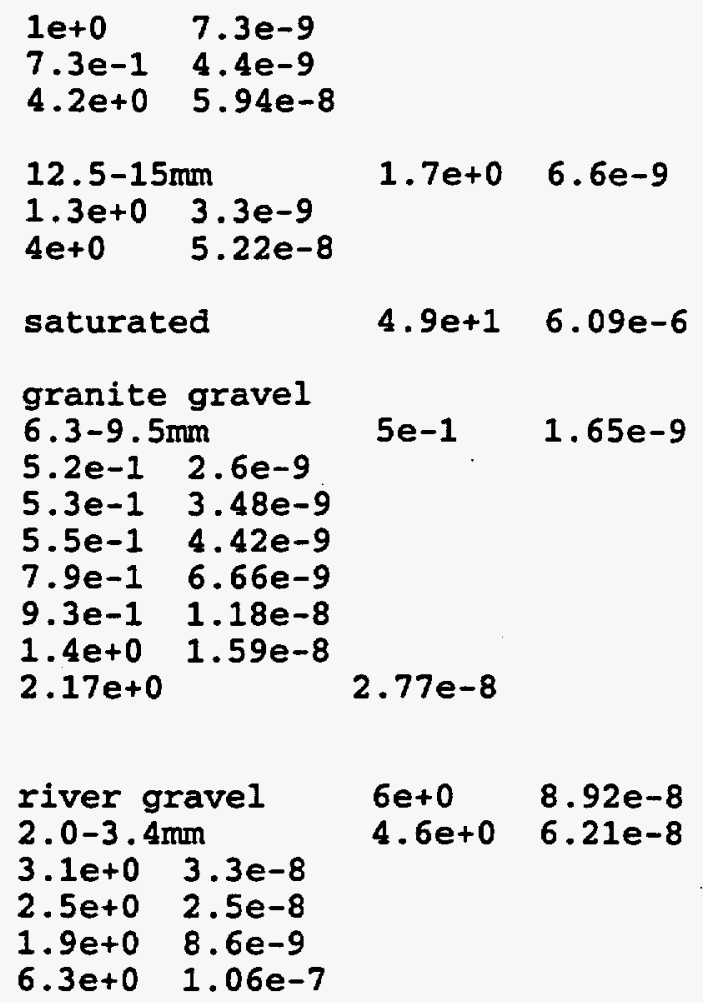

$1.091 \mathrm{e}+1$

3. $62 \mathrm{e}-7$ 

$1.14 e+1$
$1.269 e+1$
$4.12 \mathrm{e}-7$
$1.436 e+1$
$4.37 e-7$
$1.652 \mathrm{e}+1$
$4.5 e-7$
$2.153 e+1$
$5.45 e-7$
$9.75 e+0$
$1.232 e-6$
$1.011 e+1$
3. $2 e-7$
$1.11 e+1$
$1.227 e+1$
3. $62 e-7$
$1.482 \mathrm{e}+1$
4. $22 \mathrm{e}-7$
$5.05 e-7$
$6.54 e-7$
$1.937 \mathrm{e}+1$
$9.89 e-7$
$8.35 e+0$
$2.15 e-7$

Kunigel V1 Bentonite

various compaction

$2.08 e+1$

$3.58 e-6$

$3.38 e+1$

$3.58 \mathrm{e}+1$

2.53e+1

$3.17 e+1$

$2.039 e+1$

$2.57 e+1$

$4.344 \mathrm{e}+1$

$3.932 e+1$

$3.953 e+1$

unc. bentonite

density $=1.21$

Calico Hills

whole rock

benton: basalt

$25: 75$

density $=1.64$

$\mathrm{Cl}$

bentonite TC

density $=2.00$

benton/sand

density $=2.00$

$\begin{array}{lll}\text { Cs } & 1.3 e+1 & 5 e-8 \\ \mathrm{~Np} & 1.3 e+1 & 4 e-8 \\ \mathrm{U} & 1.3 e+1 & 1.6 e-8 \\ \text { Am } & 1.3 e+1 & 3 e-9\end{array}$
- $9.25904 e-6$
$1.02032 e-5$
$1.3608 e-5$
$1.1312 e-5$

$1.344 \mathrm{e}-5$

$1.568 \mathrm{e}-5$

$1.232 e-5$

$6.63 e+1 \quad 1.83 e-5$

$3.09 e+1$

$2.85 e+1$

$1.51 e-6$

$1 e-6$

4. $25 e+1$

$3.22 e-6$

Japanese cores
tuff $1.34 \mathrm{~g} / \mathrm{cc}$
$4.196 e+1$
$5.8 e-6$

basalt $2.90 \mathrm{~g} / \mathrm{cc}$

$9.49 e-1$

$2.5 e-8$

mudstone $2.72 \mathrm{~g} / \mathrm{cc}$

$1.6 e-1 \quad 4.31 e-9$

granite $2.60 \mathrm{~g} / \mathrm{cc}$

8.19e-1

$8 \cdot 3 e-9$ 


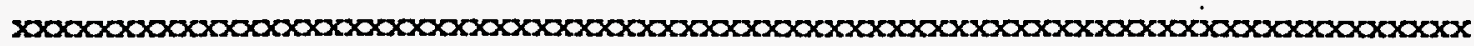
Data Set 2

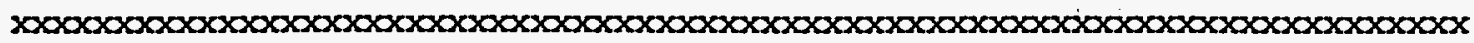

$17.32 \quad .027552 \quad 1.97$

$33.800 \quad .1344 \quad 1.75$

$\begin{array}{llll}35.8 & .1568 \quad 1.86\end{array}$

$25.3 \quad .05824 \quad 1.31$

$31.7 \quad .1232 \quad 1.64$

$\begin{array}{lll}15.92 & .020832 \quad 1.67\end{array}$

$\begin{array}{llll}17.27 & .025648 & 1.81\end{array}$

$18.19 .0336336 \quad 1.91$

$18.54 \quad .034384 \quad 1.94$

$19.6 .041944 \quad 2.05$

$20.39 \quad .0419442 .13$

$14.24 \quad 0138321.49$

$11.65 \quad .0058957 \quad 1.22$

$14.54 \quad .020272 \quad 1.53$

$17.11 \quad .035952 \quad 1.81$

$18.85 \quad .046816 \quad 1.99$

$12.05 \quad .009221 \quad 1.71$

14.18 .02191842 .05

$\begin{array}{lll}11.96 & .0088805 & 1.7\end{array}$

$13 \quad 010368 \quad 1.84$

$14.06 \quad .01828962$

25.7 .09259041 .42

$43.44 \quad .102032 \quad 1.6$

$\begin{array}{lll}39.32 & .13608 & 1.69\end{array}$

$\begin{array}{llll}39.53 & .11312 & 1.7\end{array}$

$.87 .0000529 \quad 1.74$

.945 .0001511 .89

14.9 .02122 .03

$13.44 \quad .0151088 \quad 1.83$

$11.91 \quad .01001391 .62$

11.73 .01212

$\begin{array}{llll}10.48 & .009447 & 1.79\end{array}$ 


\begin{tabular}{|c|c|c|c|c|c|c|c|c|c|c|c|}
\hline $\begin{array}{l}\text { PWBS Element: } \\
\text { Product: } \\
\text { Control Account: }\end{array}$ & $\begin{array}{l}1.2 .5 .4 \\
1122 \\
12541122\end{array}$ & & $\begin{array}{l}\text { Pert } \\
\text { TSPI } \\
\text { TS }\end{array}$ & $\begin{array}{l}\text { rmance I } \\
\text { Document } \\
\text { A Docume }\end{array}$ & $\begin{array}{l}\text { saessment } \\
\text { ation } \\
\text { ntation }\end{array}$ & & & $\begin{array}{l}\text { start D: } \\
\text { Finish D } \\
Q A-\end{array}$ & $\begin{array}{l}\text { e: } 01-0 \\
\text { te: } 18=\mathrm{m} \\
\text { YES }\end{array}$ & $\begin{array}{l}t-1997 \\
r-1999\end{array}$ & \\
\hline Annual Budget & Prior & $\begin{array}{r}\text { FY1998 } \\
948\end{array}$ & $\begin{array}{r}\text { EY1999 } \\
257\end{array}$ & $\begin{array}{r}\text { EY2000 } \\
0\end{array}$ & $\begin{array}{ccr}\text { Fiscal } & \text { Year D1stribution } \\
\text { FY2001 } & \text { FY2002 } & \text { FY2003 } \\
0 & 0 & 0\end{array}$ & $\begin{array}{r}\text { FY2004 } \\
0\end{array}$ & $\begin{array}{r}\text { FY2005 } \\
0\end{array}$ & $\begin{array}{r}\text { FY2006 } \\
0\end{array}$ & FY2007 & Future & $\begin{array}{r}\text { At } \\
\text { Complete } \\
1205\end{array}$ \\
\hline
\end{tabular}

Statement of Work

The following work shall be controlled in accordance with approved implementing procecuxes 1 dentified on the current, ocRim-accepted Requirements Traceability Network Matrix.

The approach, assumptions, models and model abstractions, scenarios, parameters, resulta, sensitivity analyaes, and conclusions and Implications for additional site characterlzation and design for TSPA-VA will be documented in a aerles of technical report suitable for external review by various types of external groups. These reports will be a comprehensive sumation of all the relevant Inputs and outputs plus discussion of the degree of conservatism in the analyses. However, each document in the serles will be produced for a different audience. The three different audiences are oxpected to be the regulator, the scientific community, and the public. Different degrees of detall and 1llustration will be developed as appropriate for each audience. Documentation may be included in the form of hardcopy or electronic files, sultable for distribution on the Whi. Some of the sectlons of this document shall be prepared in other activities (in particular the abstraction/testing activities, the scenario sections of this document shall be prepared in other activities (in particular the abstraction/testing activities, the scenario
identification and screening activity, and the TSPA -VA software upgrade and documentation activity). The Work Packages are as follows:

Document Results for TSPA-VA

TSPA Documentation Illustration and Communication Management Integration

TECHNICAL BASELINE DOCUMENTS

Work is to be performed in accordance with the latest revision of the following technlcal documents:

Level 1 - Program:

Not Applicable

Level 2 - YMSco.

Mịned Geologlc Disposal system Requirements Document

Level 3 - WAST:

Not Appllcable

Fork Packages

hork Package

12541122M1

Title

$12541122 \mathrm{M} 2$

Document Results for TSPA-VA

TSPA-VA DOC Illustration Communtcation

\begin{tabular}{|c|c|c|}
\hline & DELIVERABLES & \\
\hline Deliv ID & Description/Completion Criteria & Due Date \\
\hline SL230AM3 & $\begin{array}{l}\text { Complete TSPA-VA Analyses Technical Basis DoC } \\
\text { Criteria - } \\
\text { Description: }\end{array}$ & 21-aug-1998 \\
\hline
\end{tabular}


12541122 TSPA Documentation (continued)

DELIVERABLES

Th1s Technical Basis document w1ll support the TSPA-VA volume by providing documentation of the use of total system

models drawn from process-level models (developed in the site-characterization, environmental and design programs)

from subsystem models developed in other PA activitles and from models developed from other data sources where required to adequately represent the total system to perform analyses of radionuclide release and ultimately the peak concentration, dose or rlsk associated with these releases to the blosphers. Results of the TSPA that feed EBS design, WP design, other design/engineering activities, and site

characterization activities will be discussed in order to aliow these programs to update process-level models (1f necessary) for input into the TSPA analysis for LA. A stand alone report, fully documented as described in the TSPA Methodology and Assumptions document and sultable for external peer review will be produced. This document will follow similar outlines to previous TSPA 1 terations, but be more ancompassing to evaluate the potential effects of alternative processes and process interactions on subsystem performance.

\section{Completion Criteria:}

Th1s deliverable shall be prepared in accordance with OCRPM approved gual1ty assurance procedures Implementing requirements of the Quallty Assurance Requirements Description. The product shall be developed on the basis of the best technical data, including both $Q$ and non- $Q$ data. The $Q$ status of data used and cited in the report ahall be approprlately noted or referenced. Within the report's Reference Section, references to data shall be used in the report shall include record Accession Numbers or Data Tracking Numbers when

Acceptancecriteria

Technical data contalned within the deliverable and not already incorporated in the Geographle Nodal Information Study and Evaluation System (GENISES) shall be submitted for incorporation into the GENISEs in accordance w1th YAP-SIII.30. Verlfication of technical data submittal compliance shall be demonstrated by Including as part of the deliverable: 1) a copy of the Technical Data Information Form generated

1dentifying the data in the Automated Technical Data Tracking system, and 2) a copy of the transmittal letter attached to the technical data transmittal to the GENISEs Administrator. This deliverable shall be processed in accordance with YAP-7.10.

S/R.AWDREWS Preparer - print name

Preparer - elgnature
S/R.SPENCE Technical Reviewer - print name $\frac{5 / 11 / 9 \theta)}{\text { Date }}$
Technical Reviewer - s1gnature $\$(14 / 98)$ $\frac{\text { S/ C.HAMn TON }}{\text { QA Reviewer - print name }}$

QA Reviewer - slgnature 